\title{
A Hierarchical Energy-Efficient Framework for Data Aggregation in Wireless Sensor Networks
}

\author{
Yuanzhu Peter Chen, Arthur L. Liestman, Member, IEEE, and Jiangchuan Liu, Member, IEEE
}

\begin{abstract}
A network of sensors can be used to obtain statebased data from the area in which they are deployed. To reduce costs, the data, sent via intermediate sensors to a sink, is often aggregated (or compressed). This compression is done by a subset of the sensors called aggregators. Since sensors are usually equipped with small and unreplenishable energy reserves, a critical issue is to strategically deploy an appropriate number of aggregators so as to minimize the amount of energy consumed by transporting and aggregating the data.

In this paper, we first study single-level aggregation and propose an Energy-Efficient Protocol for Aggregator Selection (EPAS). Then, we generalize it to an aggregation hierarchy and extend EPAS to a Hierarchical Energy-Efficient Protocol for Aggregator Selection ( $h$ EPAS). We derive the optimal number of aggregators with generalized compression and powerconsumption models, and present fully distributed algorithms for aggregator selection. Simulation results show that our algorithms significantly reduce the energy consumption for data collection in wireless sensor networks. Moreover, the algorithms do not rely on particular routing protocols, and are thus applicable to a broad spectrum of application environments.
\end{abstract}

Index Terms-Wireless sensor networks, energy-efficient, hierarchy

\section{INTRODUCTION}

A wireless sensor network is a collection of sensors interconnected by wireless communication channels. Each sensor node ${ }^{1}$ is a small device that can collect data from its surrounding area, carry out simple computations, and communicate with other sensors or with the controlling authorities of the network. Long distance communications are achieved in a multi-hop fashion. Such networks have been realized due to recent advances in micro-electro-mechanical systems and are expected to be widely used for such applications as environment monitoring, intrusion detection, and earthquake warning [1].

In many of these applications, the data to be collected is state-based, that is, it consists of measurements of ambient surroundings. Significant redundancies often exist in such data due to spatial-temporal correlations. These local redundancies

An earlier version of this paper was published in QShine'05.

Yuanzhu Peter Chen's work is supported by his University Startup Grant. He is with Memorial University of Newfoundland, Canada (yzchen@cs.mun.ca).

Arthur L. Liestman's work is supported by an NSERC Discovery grant. He is with Simon Fraser University, Canada (art@cs.sfu.ca).

Jiangchuan Liu's work was supported in part by a Canadian NSERC Discovery Grant 288325, an NSERC Research Tools and Instruments Grant, a Canada Foundation for Innovation (CFI) New Opportunities Grant, and an SFU President's Research Grant. He is with Simon Fraser University, Canada (jcliu@cs.sfu.ca).

${ }^{1}$ We assume that each sensor node has only one sensor, and hence, in the rest of this paper, sensor and sensor node are used interchangeably [1] can be removed prior to sending the over-sized raw data to the sink and draining the limited sensor energy store. This process, referred to as data aggregation or data fusion, is quite attractive as it is often infeasible or costly to replenish the batteries of the deployed sensors.

It is worth noting, however, that the amount by which the data size may be reduced by aggregation depends on the application. For example, simple statistical values such as sum, mean or deviation, can be easily aggregated into a single scalar or vector. On the other hand, a temperature map of a region would allow more limited reduction. For example, a wavelet scheme separately computes the wavelet transform for each subregion first, and then merges the resulting wavelet coefficients of subregions to obtain the wavelet transform of the entire region. Here, the number of wavelet coefficients of a subregion may increase as does the number of sensors therein.

In this paper, we investigate energy-efficient aggregator selection in wireless sensor networks. A unique feature of our study is that we consider a general compression model for data aggregation, which is more realistic than the infinite compression [2], [3], [4] allowed in previous studies. We begin by using only a single level of aggregation. With this restriction, we calculate the number of aggregators needed to minimize the amount of total energy consumed in the network. A practical Energy-efficient Protocol for Aggregator Selection (EPAS) is presented to achieve the target number of aggregators. Next, we demonstrate that multiple levels of aggregation can further reduce energy consumption. EPAS is then extended to $h$ EPAS (Hierarchical EPAS) to provide a multiple level solution. We give fully distributed algorithms for aggregator selection in the above protocols, which are applicable to a broad spectrum of state-based data collection applications in sensor networks.

The performance of these algorithms are examined by simulations. Our results demonstrate that EPAS conserves energy consumed both by the entire network and by the most heavily loaded sensors. The energy consumption can be further reduced by using $h$ EPAS. The number of levels in the hierarchy is also a critical factor, and our results provide a general guideline toward desirable settings of the aggregation levels.

The remainder of this paper is organized as follows. In Section II, we provide some background and review related work. An energy-efficient protocol for aggregator selection (EPAS) for one-level data aggregation is presented in Section III. In Section IV, we generalize EPAS to accommodate aggregation hierarchies, called $h$ EPAS. The performance of these protocols is examined in Section V. Finally, Section VI concludes the 
paper and offers some future research directions.

\section{BACKGROUND AND RELATED WORK}

Wireless sensor networks have received much attention due to the number of potential applications of this technology. Many data communication protocols have been proposed lately, such as DD [5], TAG [6], TTDD [7], GRAB [8], Pilot [9], LEACH [2], and LAF [10]. Recent surveys by Akkaya and Younis [11], by Akyildiz et al. [12], and by Tilak, AbuGhazaleh and Heinzelman [13] include information on these and other protocols. There are three types of data collection in sensor networks. Event-based data, such as intrusion detection or object tracking, is collected when an event at a particular venue within the deployment region occurs. The event is confirmed by detecting sensors using local consensus and reported to the control authority [14], [15]. Focused statebased data is collected in response to a query sent to selected sensors requesting relevant data [5], [8]. Global state-based data, such as temperature or humidity, is collected by sensors all over the deployment area and transmitted to the sink [16]. Our interest here is in global state-based data.

Bhardwaj, Garnett, and Chandrakasan [17] provided an upper bound on the lifetime of sensor networks that are engaged in event detections. In their model, the energy consumed for a node to relay (that is, to receive and transmit) a unit of data to another node at distance $d$ is denoted by $\alpha_{1}+\alpha_{2} d^{l}$, where $\alpha_{1}$ and $\alpha_{2}$ depend on the hardware implementation of the sensors and $l$ is the path attenuation exponent (usually in the range $2 \leq l \leq 4$ ). The distance from one sensor to the next that minimizes the energy consumed is the characteristic distance, denoted $d_{\text {char }}$, where $d_{\text {char }}=\sqrt[l]{\frac{\alpha_{1}}{\alpha_{2}(l-1)}}$. This value depends only on the hardware design specifications and the environment.

For state-based data collection, Heinzelman, Chandrakasan and Balakrishnan [2] presented a clustering algorithm (LEACH) to aggregate the data from sensors. In LEACH, each sensor becomes a clusterhead with a fixed probability during startup and every non-clusterhead sensor joins the cluster of a nearest clusterhead. The clusterheads act as aggregators. As clusterheads are likely to consume more energy than nonclusterheads, LEACH allows rotation of clusterhead status. Alternatively, unequal-sized clusters can be used to balance the sensor energy consumption [18]. Clustering sensors and mobile ad hoc nodes, in general, has been an intensively studied area [19], [14], [20], [21], [22], [23], [24], [25].

In contrast to clustering, the design problem of determining an appropriate number of aggregators for a given sensor network has also been considered recently. This value can be used to calculate the aggregator selection probability as in LEACH, for example. Mhatre and Rosenberg [4] consider two types of nodes: regular sensors (type 0) and more powerful sensors (type 1) that can serve as clusterheads. Their work focuses on determining the numbers of type- 1 sensors in a single aggregation level. A hierarchical clustering algorithm is proposed by Bandyopadhyay and Coyle [3] assuming infinite compression. In their model, they calculate the number of aggregators in each level for energy conservation.
Our hierarchical aggregator selection protocol ( $h$ EPAS) is motivated by these studies. In particular, Bandyopadhyay and Coyle [3] show that the use of a hierarchical structure can help conserve energy. However, we consider a more realistic compression model, and propose a general hierarchical framework to minimize the total energy consumed by both communication and aggregation. Another novelty of this work is our 2-phase aggregator selection protocol (Section III-C) to produce evenly distributed aggregators.

\section{One LeVEl AgGregation}

We begin by allowing only one level of data aggregation. Sensor nodes are partitioned into clusters, each with a clusterhead. The sensors within each cluster periodically send their data to the clusterhead. The clusterhead compresses the data collected from all members and sends the aggregated data to the sink. We first construct an ideal model, where the sensors and the aggregators are uniformly distributed over the region. Then we present a distributed algorithm, EPAS, to select the aggregators under practical constraints.

\section{A. System Model and Notations}

We first state our assumptions and introduce some notation to be used. A summary list of the notation used in this paper is given in Table I.

Consider a network of $n$ sensors uniformly distributed over a region. Many large-scale sensor networks such as environment monitoring sensors dropped from aircraft have this property [12], [1]. Route calculation is carried out during the initial setup after the aggregators (clusterheads) are selected, and the sensors send packets to their respective clusterheads using multi-hop paths (if necessary). Each hop in these paths is roughly of characteristic distance $d_{\text {char }}$ [17]. That is, each node forwards the data to a node that is approximately $d_{\text {char }}$ closer to the destination.

We assume that data collection is synchronized by cycles, where each cycle consists of a round of data collection, transmission, and aggregation. During each cycle, sensors collect data. The data generated is then sent to the aggregator as a packet of $r$ bits. Each aggregator compresses the data it receives from the sensors of its cluster and then forwards the data to the sink. We assume that, by relaying packets via hops of the characteristic distance, transporting one unit of data a distance $d$ consumes $\alpha \times d$ units of energy, where $\alpha=\frac{\alpha_{1}+\alpha_{2} d_{\text {char }}^{l}}{d_{\text {char }}}$ [17]. That is, to send one unit of data a distance one requires the sender to expend $\alpha_{2} d_{\text {char }}^{l}$ units of energy to transmit the message. The sender and receiver together use a total of $\alpha_{1}$ units of energy internally.

We use a general function $g(x)$ to represent the data compressibility at aggregators. Basically, $g(x)$ gives the data volume after compression as a function of the input data volume $x$. When $g(x)$ is a constant, this is the infinite compression assumed in many of the previous studies [2], [3]. We use $f_{a}(x)$ to denote the energy consumed by compressing $x$ data units. This is generally proportional to $x$ but need not be. Although in practice the energy consumed by compressing a unit of data may be significantly less than that consumed by 
TABLE I

LIST OF NOTATION.

\begin{tabular}{|c|c|}
\hline Symbol & Meaning \\
\hline$a$ & Radius of the network deployment region \\
\hline$a_{i}$ & Radius of a level $i$ cluster \\
\hline$b$ & Coverage radius of an aggregator \\
\hline$c$ & Data compression overhead \\
\hline$d_{\text {char }}$ & Characteristic distance, $\sqrt[l]{\frac{\alpha_{1}}{\alpha_{2}(l-1)}}$ \\
\hline$E_{a}$ & $\begin{array}{l}\text { Energy consumed by aggregating data in a single } \\
\text { cycle in single-level context }\end{array}$ \\
\hline$E_{a i}$ & $\begin{array}{l}\text { Energy consumed by aggregating data in a single } \\
\text { cycle in level } i\end{array}$ \\
\hline$E_{c i}$ & $\begin{array}{l}\text { Energy consumed by transporting data from } \\
\text { level } i \text { to level } i+1 \text { in a single cycle }\end{array}$ \\
\hline$f_{a}(\cdot)$ & Aggregation energy consumption function \\
\hline$g(\cdot)$ & Data compressibility function \\
\hline$h$ & Number of levels in the hierarchy \\
\hline$k$ & Number of aggregators in single-level context \\
\hline$k_{i}$ & Number of aggregators in level $i$ \\
\hline$l$ & Propagation loss exponent \\
\hline$n$ & Number of sensors in the network \\
\hline$r$ & Sensor data rate \\
\hline$r_{i}$ & Level $i$ aggregator data rate \\
\hline$\alpha_{1}$ & Circuit energy consumption coefficient \\
\hline$\alpha_{2}$ & $\begin{array}{l}\text { Antenna energy consumption coefficient } \\
\frac{\alpha_{1}+\alpha_{2} d_{\text {char }}^{l}}{d_{\text {char }}}\end{array}$ \\
\hline$\beta$ & Aggregation energy consumption coefficient \\
\hline$\gamma$ & Data compression ratio \\
\hline
\end{tabular}

transmitting it (see [1]), we include this cost in our model for completeness, e.g., to accommodate advanced algorithms like Wavelet compression [1].

\section{B. Optimal Number of Aggregators}

We would like to determine the number of clusterheads (aggregators) that minimizes the total energy consumed by transmitting and aggregating data under our model. For simplicity, we assume that the sensors are deployed in a circular region $A$ of radius $a$ meters with the sink located at the center of the circle. Our solution can be easily extended to accommodate other region shapes or sink locations.

Let $E_{c 0}$ denote the total energy consumed by all of the sensors sending data to their respective aggregators in a single cycle. Consider the area covered by cluster $C$ centered at $\left(x_{c}, y_{c}\right)$. The total distance that the data packets travel from all members of $C$ to $\left(x_{c}, y_{c}\right)$ is $\frac{n}{\pi a^{2}} \times$ $\iint_{(x, y) \in C} \sqrt{\left(x-x_{c}\right)^{2}+\left(y-y_{c}\right)^{2}} d x d y$, where $\frac{n}{\pi a^{2}}$ is the sensor density.

If each sensor chooses the closest aggregator as its clusterhead, the sensors essentially form a Voronoi diagram of the network region where each cluster corresponds to a Voronoi cell. For large $k$, a typical cluster can be approximated as a circle of radius $\frac{a}{\sqrt{k}}[26]^{2}$ with the aggregator at the center.

\footnotetext{
${ }^{2}$ More details on the problem of covering geometric spaces with spheres can be found in Conway and Sloane [27].
}

With this, the above expression evaluates to $\frac{2 a n}{3 k^{\frac{3}{2}}}$. Thus, after factoring in the $\alpha$ coefficient to obtain the energy consumption, the sensor data rate $r$, and summing over the $k$ aggregators, we have

$$
E_{c 0}=\frac{2 \alpha a n r}{3 k^{\frac{1}{2}}} .
$$

Let $E_{a}$ denote the total energy consumed by data aggregation in a single cycle. Since the aggregator receives data at an average rate of $\frac{n r}{k}$ bits per cycle, we have

$$
E_{a}=k \times f_{a}\left(\frac{n r}{k}\right) .
$$

Let $E_{c 1}$ denote the total energy consumed by all of the aggregators sending this data to the sink in a single cycle. Since the data is sent by an aggregator at a rate of $g\left(\frac{n r}{k}\right)$ bits per cycle and the aggregator density is $\frac{k}{\pi a^{2}}$, we have $E_{c 1}=$ $g\left(\frac{n r}{k}\right) \times \alpha \times \frac{k}{\pi a^{2}} \times \iint_{(x, y) \in A} \sqrt{x^{2}+y^{2}} d x d y$, which evaluates to

$$
E_{c 1}=g\left(\frac{n r}{k}\right) \times \frac{2 k \alpha a}{3} .
$$

Summing up Equations (1), (2) and (3), we have

$$
\frac{2 \alpha a n r}{3 k^{\frac{1}{2}}}+k \times f_{a}\left(\frac{n r}{k}\right)+g\left(\frac{n r}{k}\right) \times \frac{2 k \alpha a}{3} .
$$

Consider a typical circuit power-consumption model where the aggregation energy consumption is proportional to the volume of the data to be compressed, that is, $f_{a}(x)=\beta x$, for some constant $\beta$. Also consider a typical linear compression model, $g(x)=\gamma x+c$, where $\gamma(0 \leq \gamma \leq 1)$ is the compression ratio and $c$ is the compression overhead [28]. It follows that the number of aggregators that minimizes the energy consumption of the network in a single cycle (that is, Equation (4)), is $k=\left(\frac{n r}{2 c}\right)^{\frac{2}{3}}$. Substituting $g(x)=\gamma x+c$ into Equation (3), we see that the energy consumed by transporting the data from $k$ aggregators to the sink is proportional to $n r \gamma+c k$. Thus, the contribution of $\gamma$ to the value of Equation (3) (and (4)) is independent of $k$.

Thus, to minimize energy consumption, there should be $\left(\frac{n r}{2 c}\right)^{\frac{2}{3}}$ aggregators. This conclusion also applies to the special case of $\beta=0$, that is, where the energy required to compress data is negligible (as assumed in many existing sensor systems [1]).

\section{Distributed Aggregator Selection - EPAS}

In this section, we propose a practical Energy-Efficient Protocol for Aggregator Selection (EPAS) that follows our optimal solution in the previous subsection.

EPAS is a randomized and fully distributed algorithm that consists of two phases. In the first phase, each sensor chooses to be a clusterhead (aggregator) with probability $p_{1}$ independently for some $p_{1} \in\left[0, \frac{k}{n}\right]$. Suppose that each clusterhead has a fixed coverage radius of $b$ meters. (See Section III-D for discussion of determining the value of $b$.) In the second phase, each sensor that is not within the coverage radius of some clusterhead declares itself to be a clusterhead with probability $p_{2}$. 
By careful choice of $p_{1}$ and $p_{2}$, we can ensure that the expected number of aggregators is $k$. To do that, we leverage the following propositions.

Lemma 3.1: After phase 1 of EPAS, the probability that a sensor $c$ is not covered is $\left(1-p_{1}\right)\left(1-\frac{p_{1} b^{2}}{a^{2}}\right)^{n-1}$, where $p_{1}$ is the phase- 1 selection probability, and $b$ and $a$ are the coverage and network region radii, respectively.

Proof: Let $X$ be a random variable denoting the number of sensors other than $c$ that are contained in a circle of radius $b$ centered at $c$. Thus, we have $\operatorname{Prob}[X=$ $i]=\left(\begin{array}{c}n \\ i\end{array}\right)\left(\frac{b^{2}}{a^{2}}\right)^{i}\left(1-\frac{b^{2}}{a^{2}}\right)^{n-i-1}$, for $i=0,1, \ldots, n-1$. Further, $\left(1-p_{1}\right)^{X}$ is a random variable indicating the probability that $c$ is not covered by any selected clusterhead after phase 1 . Its expected value is

$$
\begin{aligned}
E\left[\left(1-p_{1}\right)^{X}\right] & =\sum_{i=0}^{n}\left(1-p_{1}\right)^{i} \times \operatorname{Prob}[X=i] \\
& =\sum_{i=0}^{n}\left(1-p_{1}\right)^{i}\left(\begin{array}{c}
n \\
i
\end{array}\right)\left(\frac{b^{2}}{a^{2}}\right)^{i}\left(1-\frac{b^{2}}{a^{2}}\right)^{n-i-1} \\
& =\left(1-\frac{p_{1} b^{2}}{a^{2}}\right)^{n-1} .
\end{aligned}
$$

This is also the probability that $c$ is not covered by any selected clusterhead. Factoring in the probability that $c$ does not select itself, we have the expected probability that sensor $c$ is not covered: $\left(1-p_{1}\right)\left(1-\frac{p_{1} b^{2}}{a^{2}}\right)^{n-1}$.

For the expected number of clusterheads generated by EPAS to be $k$, the selection probabilities of the two phases should satisfy the following condition.

Theorem 3.2: The expected number of clusterheads generated by EPAS is $k$ iff $p_{1}$ and $p_{2}$ are chosen such that $p_{1}+p_{2}\left(1-p_{1}\right)\left(1-\frac{p_{1} b^{2}}{a^{2}}\right)^{n-1}=\frac{k}{n}$.

Proof: Let random variable $Y$ denote the number of clusterheads generated by EPAS. Let binary random variable $Y_{i}$ be 1 if and only if sensor $c_{i}(i=1,2, \ldots, n)$ becomes a clusterhead. Thus we have $E\left[Y_{i}\right]=\frac{E[Y]}{n}=\frac{k}{n}$. Let binary random variables $Y_{i, 1}$ and $Y_{i, 2}$ denote the fact that $c_{i}$ becomes a clusterhead in phases 1 and 2, respectively. Since the events that $c_{i}$ becomes a clusterhead in either phase are mutually exclusive, we have

$$
\begin{aligned}
E\left[Y_{i}\right] & =\operatorname{Prob}\left[Y_{i}=1\right] \\
& =\operatorname{Prob}\left[\left(Y_{i, 1}=1\right) \cup\left(Y_{i, 2}=1\right)\right] \\
& =\operatorname{Prob}\left[Y_{i, 1}=1\right]+\operatorname{Prob}\left[Y_{i, 2}=1\right] \\
& =E\left[Y_{i, 1}\right]+E\left[Y_{i, 2}\right] .
\end{aligned}
$$

We know that $E\left[Y_{i, 1}\right]=p_{1}$. In addition, we have $E\left[Y_{i, 2}\right]=p_{2} \times \operatorname{Prob}[c$ is not covered in phase 1]. Based on Lemma 3.1, we have $\operatorname{Prob}[c$ is not covered in phase 1] = $\left(1-p_{1}\right)\left(1-\frac{p_{1} b^{2}}{a^{2}}\right)^{n-1}$. As a result, we have $\frac{k}{n}=E\left[Y_{i, 1}\right]+$ $E\left[Y_{i, 2}\right]=p_{1}+p_{2}\left(1-p_{1}\right)\left(1-\frac{p_{1} b^{2}}{a^{2}}\right)^{n-1}$.

After phase 2, the expected number of aggregators is $k$. Each sensor that is not an aggregator selects the closest aggregator as its clusterhead. Thus, the clusters essentially form a Voronoi partitioning of the network.

\section{Discussion}

Given the number of sensors, deployment area, compression ratio, characteristic distance, and other network parameters, we can calculate the optimal number of aggregators $k$. Given this target number of aggregators, we can choose appropriate probabilities $p_{1}$ and $p_{2}$ offline. After deployment, the sensors select $k$ aggregators. Each of the aggregators broadcasts its status to all sensors within coverage radius $b$. For large $k, k$ circles of radius $b=\sqrt{\frac{3 \sqrt{3}}{2 \pi}} \frac{a}{\sqrt{k}} \approx 1.0996 \times \frac{a}{\sqrt{k}}$ can cover the entire region of area $\pi a^{2}$ [26]. We use a larger coverage radius $b=\frac{2 a}{\sqrt{k}}$ to ensure that most of the sensors are within the coverage radius of at least one aggregator while keeping the broadcast radius small. In Section V, we report on a set of experiments to determine how many sensors lie outside the coverage areas for $k$ aggregators with coverage radius $b=\frac{2 a}{\sqrt{k}}$.

\section{Hierarchical Aggregation}

We now consider a more general framework that organizes the aggregators in a hierarchy. We begin with all sensors in level 0 of the hierarchy. From those sensors, we select a subset as aggregators for level 1. From the level 1 aggregators, we select a subset to act as level 2 aggregators. Similarly, we select a subset of the aggregators at each level to act as aggregators at the next higher level. Finally, the sink (which may not be an aggregator of any of the other levels) is the only aggregator of level $h+1$.

Once the aggregation hierarchy is established, sensors collect data and send it to the nearest level 1 aggregator. The level 1 aggregators collect this data from their sensors, aggregate it, and forward it to the nearest level 2 aggregator. This process continues until the level $h$ aggregators forward the data to the sink.

In this section, we modify the method of Section III to determine the optimal number of aggregators in each level of the hierarchy. Then we extend EPAS to $h$ EPAS, its hierarchical version.

\section{A. Optimal numbers of aggregators in the hierarchy}

We denote the number of aggregators in level $i$ by $k_{i}(i=$ $0,1, \ldots, h+1)$. Note that $k_{0}=n$ and $k_{h+1}=1$. The data is sent out of a level $i$ aggregator to its clusterhead at a rate of $r_{i}$ bits/cycle and $r_{0}=r$. As before, the data compression rate is described by function $g(\cdot)$.We assume that aggregators in each level are distributed uniformly. That is, a level $i$ aggregator receives data from $\frac{k_{i-1}}{k_{i}}$ level $(i-1)$ aggregators. The data rate $r_{i}$ can be expressed as:

$$
r_{i}= \begin{cases}r & \text { if } i=0 \\ g\left(r_{i-1} \times \frac{k_{i-1}}{k_{i}}\right) & i=1,2, \ldots, h .\end{cases}
$$

Let $E_{a i}$ be the total energy consumed by the compression done by all of the aggregators of each level $i$ in a single cycle. We have $E_{a i}=k_{i} \times f_{a}\left(\frac{k_{i-1}}{k_{i}} \times r_{i-1}\right)$.

We now consider $E_{c i}$, the total energy consumed by transporting data from level $i$ aggregators to level $(i+1)$ 
aggregators $(i=0,1, \ldots, h)$ in a single cycle. As before, a typical level $(i+1)$ cluster $C$ can be approximated with a circle of radius $a_{i+1}=a / \sqrt{k_{i+1}}$, centered at $\left(x_{c}, y_{c}\right)$, and the density of the level $i$ aggregators is $\frac{k_{i}}{\pi a^{2}}$. The portion of $E_{c i}$ within this cluster is $r_{i} \times \alpha \times$ $\frac{k_{i}}{\pi a^{2}} \times \iint_{(x, y) \in C} \sqrt{\left(x-x_{c}\right)^{2}+\left(y-y_{c}\right)^{2}} d x d y=\frac{2 \alpha a k_{i} r_{i}}{3 k_{i+1}^{\frac{3}{2}}}$.

Therefore, summing over the $k_{i+1}$ level $(i+1)$ clusters, we have $E_{c i}=\frac{2 \alpha a k_{i} r_{i}}{3 k_{i}^{\frac{1}{2}}}$.

Thus, the total energy consumed in a single cycle is

$$
\sum_{i=1}^{h} E_{a i}+\sum_{i=0}^{h} E_{c i},
$$

which is a function of the $k_{i}$.

Given values of $r, a, \alpha, \gamma$ and $c$ for a particular system, the values of $k_{i}$ minimizing the above total energy consumption can be calculated. These values can then be used to configure the aggregator selection protocol.

\section{B. Hierarchical EPAS}

Here, we propose a Hierarchical Energy-Efficient Protocol for Aggregator Selection ( $h \mathrm{EPAS}$ ). We assume that the optimal number of aggregators in each level $i$ is $k_{i}(i=1,2, \ldots, h)$ as calculated above. The protocol selects an expected $k_{i}$ sensors as the level $i$ aggregators. $h$ EPAS executes for $h$ iterations. Each iteration is similar to EPAS (Section III-C). During iteration $i$, a level $(i-1)$ aggregator chooses to become a level $i$ aggregator with probability $p_{i} \in\left[0, \frac{k_{i}}{n}\right]$ in the first phase. Each chosen aggregator has a coverage radius of $b=\frac{2 a}{\sqrt{k_{i}}}$. In the second phase, a level $(i-1)$ aggregator that is not covered by any level $i$ aggregator chooses to become a level $i$ aggregator with probability $p_{2}$, where $p_{1}$ and $p_{2}$ satisfy the condition described in Theorem 3.2 for $k=k_{i}$. After the aggregators of all levels are chosen, each level $i$ aggregator $(i=0,1, \ldots, h)$ joins the cluster of the nearest level $(i+1)$ aggregator.

\section{Performance Evaluation}

We evaluate the performance of EPAS and $h$ EPAS through simulations using our custom simulator. We first investigate the energy consumption versus the number of aggregators in the single-level case in order to understand the effectiveness of EPAS. Then we study the energy saving gained by adding more levels to the aggregation hierarchy.

In the simulation, we use a network of 10000 sensors uniformly deployed in a circular region of radius 1000 meters. The system specifications we use are similar to those used by Heinzelman, Chandrakasan and Balakrishnan [2] and by Mhatre and Rosenberg [4] (see Table II). We assume that the sensors sample the environment every minute. This differs slightly from the assumptions in these previous paper but is reasonable for our application. The measured values are converted to 16-bit digital representations and a single cycle lasts for 10 minutes. Therefore, the sensor data rate is 160 bits/cycle.

The regions served by the aggregators of each level form a Voronoi diagram of the overall network region. In the single
TABLE II

SYSTEM SPECIFICATIONS

\begin{tabular}{r|l} 
Parameter & Value \\
\hline \hline Number of sensors $n$ & 10000 \\
Network radius a & $1000 \mathrm{~m}$ \\
Communication circuit power $\alpha_{1}$ & $5 \times 10^{-8} \mathrm{~J} / \mathrm{bit}$ \\
Communication antenna power $\alpha_{2}$ & $1 \times 10^{-10} \mathrm{~J} / \mathrm{bit}_{\mathrm{m}}{ }^{2}$ \\
Propagation loss exponent $l$ & 2 \\
Characteristic distance $d_{\text {char }}$ & $22.36 \mathrm{~m}$ \\
Aggregation energy consumption rate $\beta$ & $5 \times 10^{-8} \mathrm{~J} / \mathrm{bit}$ \\
Sensor data rate $r$ & $160 \mathrm{bits} / \mathrm{sec}$ \\
Data compression ratio $\gamma$ & $25 \%$ \\
Data compression overhead $c$ & $32 \mathrm{bits}$
\end{tabular}

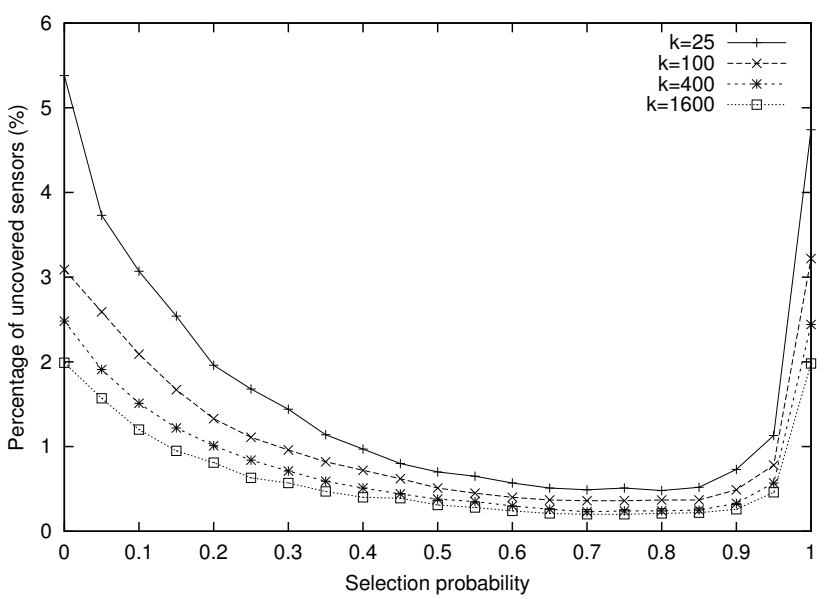

Fig. 1. Uncovered sensors vs phase-1 probability.

level EPAS protocol, each aggregator compresses the data collected from sensors located within its Voronoi cell and sends it to the sink at the center of the region. To mitigate the impact of inefficient routing, we assume an idealized routing protocol, Characteristic Distance Progressive Routing (CDPR), to approximate straight-line routing [17]. In CDPR, when a packet intended for node $d$ is at node $s$ with $\operatorname{dist}(s, d)>$ $d_{\text {char }}$, it is forwarded to an intermediate node $v_{1}$ that is the closest to the target point $t$, a point on the line segment $(s, d)$ at distance $d_{\text {char }}$ from $s$. The packet is forwarded one step at a time until it reaches node $v_{i}$ within distance $d_{\text {char }}$ of $d$ from which it is forwarded to $d$ directly.

Our next goal is to choose a suitable value of $p_{1}$, and thus that of the corresponding $p_{2}$, that leaves few sensors uncovered after EPAS. To do this, we fix $k$ and $n$, select a coverage radius $b$, and then, using various appropriate values of $p_{1}$, simulate the placement of the sensors and the choice of aggregators using that value of $p_{1}$. For each simulation, we count the number of uncovered sensors. In particular, we then assume that there are 10000 sensors and we choose the expected numbers of aggregators $k$ to be $25,100,400$, or 1600. Having fixed $k$, we use the coverage radius $b=\frac{2 \times 1000}{\sqrt{k}}$. For the above values of $k$, this gives $b=400,200,100$, and 50 meters, respectively. We consider each value of $p_{1}$ such that $p_{1} /(k / n)=0.05 i$, where $i=0,1,2, \ldots, 20$. 

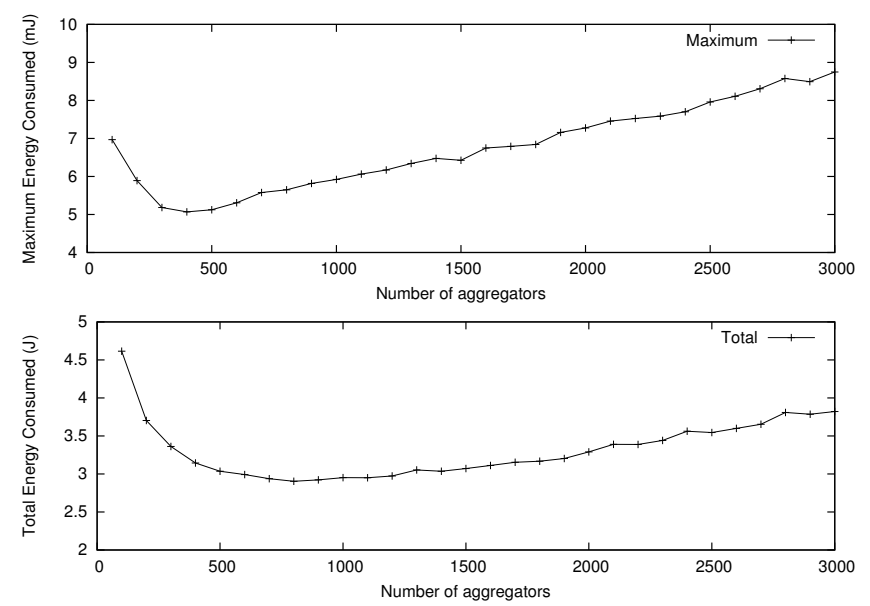

Fig. 2. Single-level energy consumption.

Figure 1 shows the percentage of sensors not covered by any aggregator for each choice of $p_{1} /(k / n)$. That is, the percentage of sensors not covered by any aggregator varies as $p_{1}$ does. Moreover, the fewer uncovered sensors, the better an aggregator's Voronoi cell can be approximated by a circle. Thus, choosing $p_{1} /(k / n) \in[0.7,0.8]$ should yield the fewest uncovered sensors. For subsequent simulations, we use 0.75 as the value for $p_{1} /(k / n)$ and obtain the value of $p_{2}$ from Theorem 3.2.

We now measure the energy consumed in a single cycle of the data collection assuming a single level of aggregation. We fix the number of aggregators, select them, and then simulate the collection of data at each sensor, transmission of the data to the aggregators, aggregation of the data, and transmission of the aggregated data to the sink. For each simulation, we compute the maximum energy consumed by an individual sensor in the network. That is, if the sensor is not an aggregator, we count the energy needed to transmit its data to the aggregator and any energy used to forward other data during the cycle. For aggregators, we also include the cost of aggregation. In addition to determining the maximum of these costs, we also compute the total of these costs over all sensors. In the simulations, we again assume that we have 10000 sensors. We choose the number of aggregators to be a value $100 j$, where $j=1,2, \ldots, 30$. Figure 2 shows the resulting maximum energy consumption and total energy consumption values. The total energy consumption appears to be minimized when the number of aggregators is between 800 and 900 . Note that the value predicted by Equation (3) is 855. The maximum energy consumption is minimized when the number of aggregators is between 450 and 500 and is reasonably small for the range between 300 and 1000. Our simulations show that these most heavily loaded nodes are generally located near the aggregators or the sink. Such nodes are required to transport large amounts of data since the data is being concentrated at the aggregators and the sink.

We now consider whether additional energy savings can be achieved by instituting a hierarchical structure for the aggregators using the $h$ EPAS protocol as described above. In an $h$-level hierarchy, we have calculated the number of
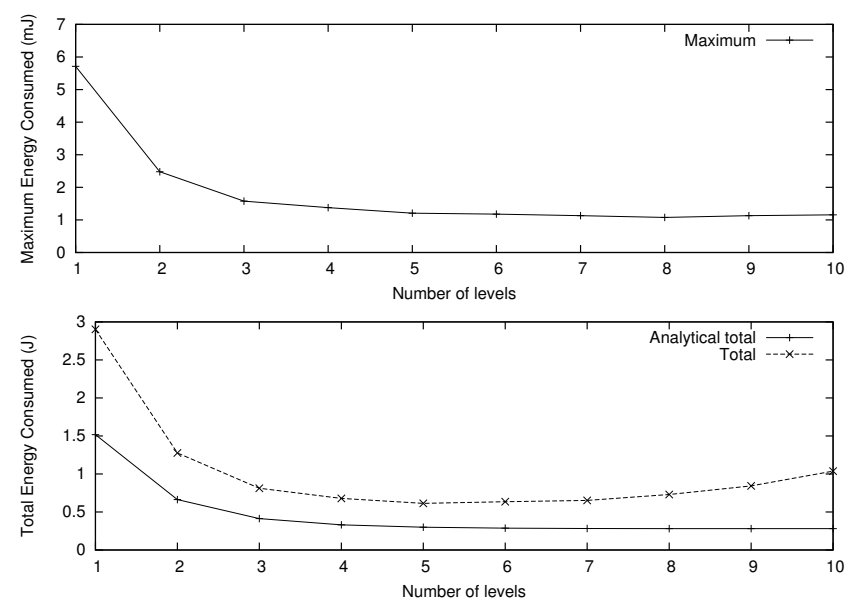

Fig. 3. Hierarchical energy consumption.

aggregators $k_{i}$ at each level $i$ of the hierarchy that minimize the total energy consumption in an ideal situation. This total is given by Equation (5) and we can calculate the values of $k_{i}$ that achieve this minimum. Table III shows the values of $k_{i}$ for $h$ levels where $1 \leq h \leq 10$ with a total of 10000 sensors deployed. We performed some simulations for $h$-level hierarchies where $1 \leq h \leq 10$ using the computed values $k_{i}$ shown in the table. In each simulation, we used the $h$ EPAS protocol to select $k_{i}$ aggregators at each level $i$ and then measured the energy consumed by each sensor, recording both the maximum for any sensor and the total consumed by all sensors. For each choice of $h$, we performed 1000 simulations. The averages of these values over all of the simulations for each $h$ are shown in the two plots in Figure 3. The ideal values from Equation (5) are included in the lower plot (which shows the total energy consumed). From these experimental results, we conclude that for 10000 sensors, the best choice would be to use a hierarchical solution with 5 levels. Note that the experimental results are reasonably close to the ideal values, which indicates that our ideal scenario is a good model of the actual situation.

\section{CONCLUSION}

In this paper, we studied the energy consumed in wireless sensor networks in which some sensors can aggregate the data. In particular, we considered one scenario that allowed a single level of aggregation assuming a general compressibility function. we calculated the number of aggregators needed to minimize the amount of total energy consumed in the network. A practical Energy-efficient Protocol for Aggregator Selection (EPAS) was presented to achieve the target number of aggregators.

We then considered a more general scenario with multiple levels of aggregation, and extended EPAS to its hierarchical version, $h$ EPAS. We gave fully distributed algorithms for aggregator selection in the above protocols, which are applicable to a broad spectrum of state-based data collection applications in sensor networks.

We performed a series of simulations, measuring energy consumption in networks with different numbers of levels 
TABLE III

NUMBER OF AGGREGATORS.

\begin{tabular}{r|rrrrrrrrrr|r}
\multicolumn{1}{c|}{$k_{1}$} & $k_{2}$ & $k_{3}$ & $k_{4}$ & $k_{5}$ & $k_{6}$ & $k_{7}$ & $k_{8}$ & $k_{9}$ & $k_{10}$ & $E_{t}$ \\
\hline \hline 1 & 855.0 & & & & & & & & & & 1.5174 \\
2 & 1848.8 & 369.8 & & & & & & & & & 0.6626 \\
3 & 3210.9 & 808.4 & 177.7 & & & & & & & & 0.4134 \\
4 & 4478.2 & 1411.2 & 387.8 & 93.3 & & & & & & & 0.3306 \\
5 & 5281.6 & 1953.8 & 659.4 & 198.3 & 52.6 & & & & & & 0.2999 \\
6 & 5689.9 & 2299.2 & 890.4 & 321.8 & 106.0 & 31.2 & & & & & 0.2878 \\
7 & 5878.2 & 2478.7 & 1033.9 & 419.4 & 162.1 & 58.5 & 19.3 & & & & 0.2829 \\
8 & 5972.7 & 2565.3 & 1108.8 & 477.8 & 202.9 & 83.7 & 33.0 & 12.2 & & & 0.2810 \\
9 & 5998.3 & 2600.7 & 1142.6 & 506.5 & 225.3 & 101.0 & 43.9 & 18.9 & 7.9 & & 0.2804 \\
10 & 6005.8 & 2608.4 & 1149.8 & 512.8 & 230.4 & 107.4 & 48.2 & 22.5 & 10.6 & 5.1 & 0.2803
\end{tabular}

and aggregators at each level. Overall, our simulations show that both the total energy consumption and the maximum energy consumption among sensors are significantly reduced by employing the proposed protocols.

This work can be extended in a number of different ways. For example, we can better balance the energy consumption among nodes by the use of mobile aggregators. We may also consider the problem of aggregator selection for sensor networks with heterogeneous nodes.

\section{REFERENCES}

[1] F. Zhao and L. Guibas, Wireless Sensor Networks : An Information Processing Approach (Morgan Kaufmann Series in Networking). Morgan Kaufmann, 2004.

[2] W. B. Heinzelman, A. Chandrakasan, and H. Balakrishnan, "An application-specific protocol architecture for wireless microsensor networks," IEEE Transactions on Wireless Communications, vol. 1, no. 4, pp. 660-670, October 2002 .

[3] S. Bandyopadhyay and E. J. Coyle, "An energy efficient hierarchical clustering algorithm for wireless sensor networks," in Proceedings of INFOCOM, San Francisco, CA, March 2003, pp. 1713-1723.

[4] V. Mhatre and C. Rosenberg, "Design guidelines for wireless sensor networks: Communication, clustering and aggregation," Ad Hoc Networks, vol. 2, no. 1, pp. 45-63, January 2004.

[5] C. Intanagonwiwat, R. Govindan, D. Estrin, J. S. Heidemann, and F. Silva, "Directed diffusion for wireless sensor networking," IEEE/ACM Transactions on Networking, vol. 11, no. 1, pp. 2-16, February 2003.

[6] S. Madden, M. J. Franklin, J. M. Hellerstein, and W. Hong, "TAG: A tiny aggregation service for ad-hoc sensor networks," in Proceedings of the 5th Symposium on Operating System Design and Implementation (OSDI), Boston, MA, December 2002, pp. 131-146.

[7] F. Ye, H. Luo, J. Cheng, S. Lu, and L. Zhang, "A two-tier data dissemination model for large-scale wireless sensor networks," in Proceedings of MobiCom, Atlanta, GA, September 2002, pp. 148-159.

[8] F. Ye, G. Zhong, S. Lu, and L. Zhang, "Robust data delivery protocol for large scale sensor networks," in Proceedings of IEEE International Workshop on Information Processing in Sensor Networks (IPSN), Palo Alto, CA, April 2003, pp. 658-673.

[9] J. Luo, P. T. Eugster, and J.-P. Hubaux, "Pilot: Probabilistic lightweight group communication system for ad hoc networks," IEEE Transactions on Mobile Computing, vol. 3, no. 2, pp. 164-179, April-June 2004.

[10] H. Sabbineni and K. Chakrabarty, "Location-aided flooding: An energyefficient data dissemination protocol for wireless sensor networks," IEEE Transactions on Computers, vol. 51, no. 1, pp. 36-46, January 2005.

[11] K. Akkaya and M. Younis, "A survey on routing protocols for wireless sensor networks," Ad Hoc Networks, vol. 3, no. 3, pp. 325-349, May 2005.

[12] I. F. Akyildiz, W. Su, Y. Sankarasubramaniam, and E. Cayirci, "Wireless sensor networks: a survey," Computer Networks, vol. 38, no. 4, pp. 393 422, March 2002
[13] S. Tilak, N. B. Abu-Ghazaleh, and W. B. Heinzelman, "A taxonomy of wireless micro-sensor network models," ACM SIGMOBILE Mobile Computing and Communications Review, vol. 6, no. 2, pp. 28-36, April 2002.

[14] W.-P. Chen, J. C. Hou, and L. Sha, "Dynamic clustering for acoustic target tracking in wireless sensor networks," IEEE Transactions on Mobile Computing, vol. 3, no. 3, pp. 258-271, July-September 2004.

[15] G. J. Pottie and W. J. Kaiser, "Wireless integrated network sensors," Communications of the ACM, vol. 43, no. 5, pp. 51-58, May 2000.

[16] D. Marco and D. L. Neuhoff, "Reliability vs. efficiency in distributed source coding for field-gathering," in Proceedings of Information Processing in Sensor Networks (IPSN), Berkeley, CA, April 2004.

[17] M. Bhardwaj, T. Garnett, and A. Chandrakasan, "Upper bounds on the lifetime of sensor networks," in Proceedings of IEEE International Conference on Communications, vol. 3, Helsinki, Finland, June 2001, pp. $785-790$.

[18] S. Soro and W. B. Heinzelman, "Prolonging the lifetime of wireless sensor networks via unequal clustering," in Proceedings of the 5th International Workshop on Algorithms for Wireless, Mobile, Ad Hoc and Sensor Networks (IEEE WMAN '05), Denver, CO, April 2005.

[19] J. Blum, M. Ding, A. Thaeler, and X. Cheng, "Connected dominating set in sensor networks and MANETs," in Handbook of Combinatorial Optimization, D.-Z. Du and P. Pardalos, Eds. Kluwer Academic Publishers, 2004.

[20] Y. P. Chen and A. L. Liestman, "Approximating minimum size weaklyconnected dominating sets for clustering mobile ad hoc networks," in The Third ACM International Symposium on Mobile Ad Hoc Networking and Computing (MobiHoc'02), Lausanne, Switzerland, June 2002, pp. $165-172$.

[21] Y. P. Chen, A. L. Liestman, and J. Liu, "Clustering algorithms for ad hoc wireless networks," in Ad Hoc and Sensor Networks, Y. Pan and Y. Xiao, Eds. Nova Science Publishers, 2004.

[22] L. Jia, R. Rajaraman, and T. Suel, "An efficient distributed algorithm for constructing small dominating sets," in Proceedings of the 20th ACM Symposium on Principles of Distributed Computing (PODC'01), 2001.

[23] J. Sucec and I. Marsic, "An energy efficient hierarchical clustering algorithm for wireless sensor networks," in Proceedings of INFOCOM, New York, NY, June 2003, pp. 1698-1706.

[24] J. Wu and W. Lou, "Extended multipoint relays to determine connected dominating sets in MANETs," in Proceedings of the First IEEE Communications Society Conference on Sensor and Ad Hoc Communications and Networks (SECON), Santa Clara, CA, 2004.

[25] O. Younis and S. Fahmy, "HEED: A hybrid, energy-efficient, distributed clustering approach for ad-hoc sensor networks," IEEE Transactions on Mobile Computing, vol. 3, no. 4, pp. 366-379, October-December 2004.

[26] R. Kerschner, "The number of circles covering a set," American Journal of Mathematics, vol. 61, pp. 665-671, 1939.

[27] J. H. Conway and N. J. A. Sloane, Sphere Packings, Lattices and Groups (Grundlehren der mathematischen Wissenschaften). Springer, 1998.

[28] J. Chou, D. Petrovic, and K. Ramchandran, "A distributed and adaptive signal processing approach to reducing energy consumption in sensor networks," in Proceedings of INFOCOM, San Francisco, CA, MarchApril 2003, pp. 1054-1062. 


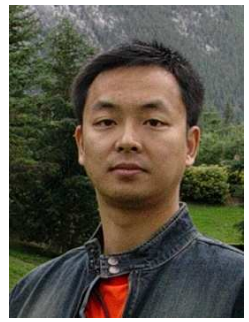

Yuanzhu Peter Chen Yuanzhu Peter Chen is an Assistant Professor in the Department of Computer Science at Memorial University of Newfoundland in St. John's, Newfoundland. He received his Ph.D. from Simon Fraser University in 2004 and B.Sc. from Peking University in 1999. Between 2004 and 2005 , he was a post-doctoral researcher at Simon Fraser University. His research interests include mobile ad hoc networking, wireless sensor networking, distributed computing, combinatorial optimization and graph theory.

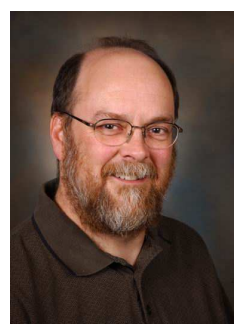

Arthur L. Liestman Arthur L. Liestman received his undergraduate degree from the University of Kansas and his M.S. and Ph.D. degrees from the University of Illinois. He is a Professor in the School of Computing Science at Simon Fraser University in Burnaby, British Columbia. His research interests involve the interplay between network communication and network structure.

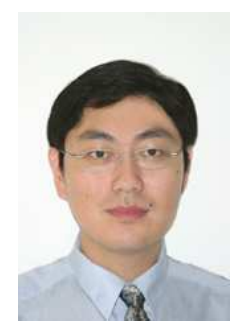

Jiangchuan Liu Jiangchuan Liu (S'01-M'03) received the B.Eng degree (cum laude) from Tsinghua University, Beijing, China, in 1999, and the Ph.D. degree from The Hong Kong University of Science and Technology in 2003, both in computer science.

He is currently an assistant professor in the School of Computing Science, Simon Fraser University, $\mathrm{BC}$, Canada, and was an assistant professor at The Chinese University of Hong Kong from 2003 to 2004. He was a recipient of Microsoft research fellowship (2000), a recipient of Hong Kong Young Scientist Award (2003), and a co-inventor of one European patents and two US patents. He won first-class honors in several regional and national programming contests.

His research interests include Internet architecture and protocols, media streaming, wireless ad hoc networks, and service overlay networks. He serves as TPC member for various networking conferences, including IEEE INFOCOM, IEEE MASS, and IWQoS. He was TPC Co-Chair for The First IEEE International Workshop on Multimedia Systems and Networking (WMSN'05), Information System Co-Chair for IEEE INFOCOM'04, and a guest-editor for ACM/Kluwer Journal of Mobile Networks and Applications (MONET), Special Issue on Energy Constraints and Lifetime Performance in Wireless Sensor Networks. He is an editor of IEEE Communications Surveys and Tutorials. He is a member of IEEE and IEEE Communications Society, and an elected member of Sigma Xi. 